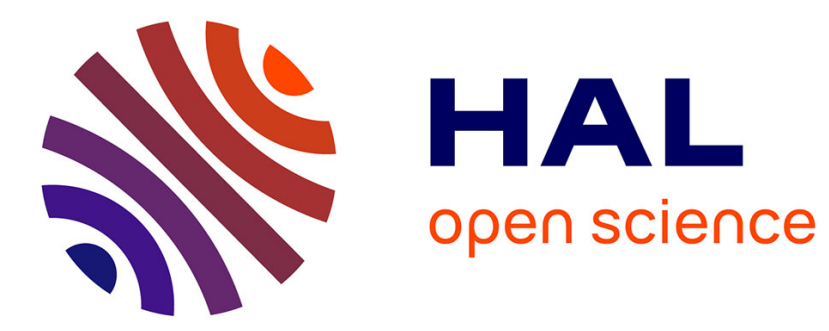

\title{
L'atelier sidérurgique gallo-romain du Latté à Oulches (Indre)
}

\author{
Nadine Dieudonné-Glad
}

\section{To cite this version:}

Nadine Dieudonné-Glad. L'atelier sidérurgique gallo-romain du Latté à Oulches (Indre). Gallia - Archéologie de la France antique, 2000, Mines et métallurgies en Gaule, 57, pp.63-75. 10.3406/galia.2000.3210 . hal-01902610

\section{HAL Id: hal-01902610 https://hal.science/hal-01902610}

Submitted on 20 Jan 2020

HAL is a multi-disciplinary open access archive for the deposit and dissemination of scientific research documents, whether they are published or not. The documents may come from teaching and research institutions in France or abroad, or from public or private research centers.
L'archive ouverte pluridisciplinaire HAL, est destinée au dépôt et à la diffusion de documents scientifiques de niveau recherche, publiés ou non, émanant des établissements d'enseignement et de recherche français ou étrangers, des laboratoires publics ou privés.

\section{(ㅇ)(1) $\$$}

Distributed under a Creative Commons Attribution - NonCommercial - NoDerivatives $\mid 4.0$ 


\title{
L'ATELIER SIDÉRURGIQUE GALLO-ROMAIN DU LATTÉ À OULCHES (INDRE)
}

\author{
Nadine DiEUdONNÉ-GLAD
}

Mots-clés. Gallo-Romain, sidérurgie, atelier, réduction, épuration, forge, fourneau, scorie, laitier, battilures.

Key-words. Roman Antiquity, iron metallurgy, workshop, smelting, smithing, furnace, slag, hammer scale.

Résumé. L'atelier métallurgique d'Oulches, en activité au IV s. après J.-C., fait partie d'un ensemble de plus de 200 sites mélallurgiques anciens répartis autour de l'agglomération antique d'Argentomagus (Indre). Sa fouille a révélé la présence de toutes les activités de la chaîne opératoire de la métallurgie du fer : traitement du minerai, réduction, épuration, forge. De plus, une phase de travail inconnue jusqu'ici a élé repérée; il s'agit de la production et du retrailement du laitier à grenaille contenant du mélal fortement carburé. Cet atelier original pourrail peut-être être mis en relation avec la fabrique d'armes citée par la Notitia Dignitatum à Argentomagus.

\begin{abstract}
The iron metallurgical workshop in Oulches is one of the very few sites in France which has revealed a series of organized structures corresponding to all stages of the iron processing in the Roman Antiquity, from ore processing and smelting to smithing. Moreover, the discovery of a wood clad pool which contained scraps of ancient blast furnaces such as slags raises the question of the deliberate production of highly carburized iron which seems to be ascerlained in Oulches. The long-lasting activity of the workshop proves that it found a place in the economic life of the area. Being in operation during the $4^{\text {th }}$ century $A D$, this activity mighl have been connected with the manufacture of weapons that the Notitia Dignitatum mentions in the small town of Argentomagus which is only $20 \mathrm{~km}$ from Oulches.
\end{abstract}

Des recherches systématiques sur la métallurgie du fer dans la civitas des Bituriges ont été entreprises en 1987 sous la forme d'une thèse soutenue en 1991 (DieudonnéGlad, 1991). Les premiers résultats obtenus en prospection ont permis de concentrer les recherches sur une zone où les vestiges de cette activité étaient particulièrement abondants, dans le cadre d'une action thématique programmée (ATP) financée par le CNRS et dirigée par F. Dumasy (université de Paris I) et dans le cadre d'une thèse sur l'occupation du sol ${ }^{86}$. Les recherches se sont poursuivies par la fouille de deux ateliers de réduction. Celui d'Oulches, exploré de 1991 à 1993, a livré les vestiges d'un atelier de la fin de l'Antiquité présentant, outre les témoins d'activités habituelles sur ce type de site

86. L. Laüt, I'espace rural autour de l'agglomération gallo-romaine d'Argentomagus, Thèse de l'univ. de Paris I, 1995.
- réduction et forge - des vestiges d'activités non encore attestées pour l'Antiquité et le Moyen Âge. La fouille proprement dite a été suivie de travaux de laboratoire concernant l'analyse élémentaire des matières premières et des scories ${ }^{87}$, mais aussi de l'étude métallographique de fragments métalliques et d'expérimentations visant à la reconstitution de certains procédés mis en évidence pour la première fois ${ }^{88}$.

87. Ces analyses ont été effectuées sous la responsabilité d'Alain Ploquin dans le cadre du programme PALSID au Centre de recherches pétrographiques et géochimiques (CNRS) de Vandœuvrelès-Nancy. Nous tenons à le remercier pour l'aide qu'il nous a apportée dans l'interprétation des résultats.

88. Ces travaux ont été menés en collaboration avec J. Parisot (CNRS) au Laboratoire de mécanique et de physique des matériaux de l'École nationale supérieure de mécanique et d'aérotechnique de Poitiers, dans le cadre de travaux de laboratoires effectués par J.-M. Charmillon, élève ingénieur. 


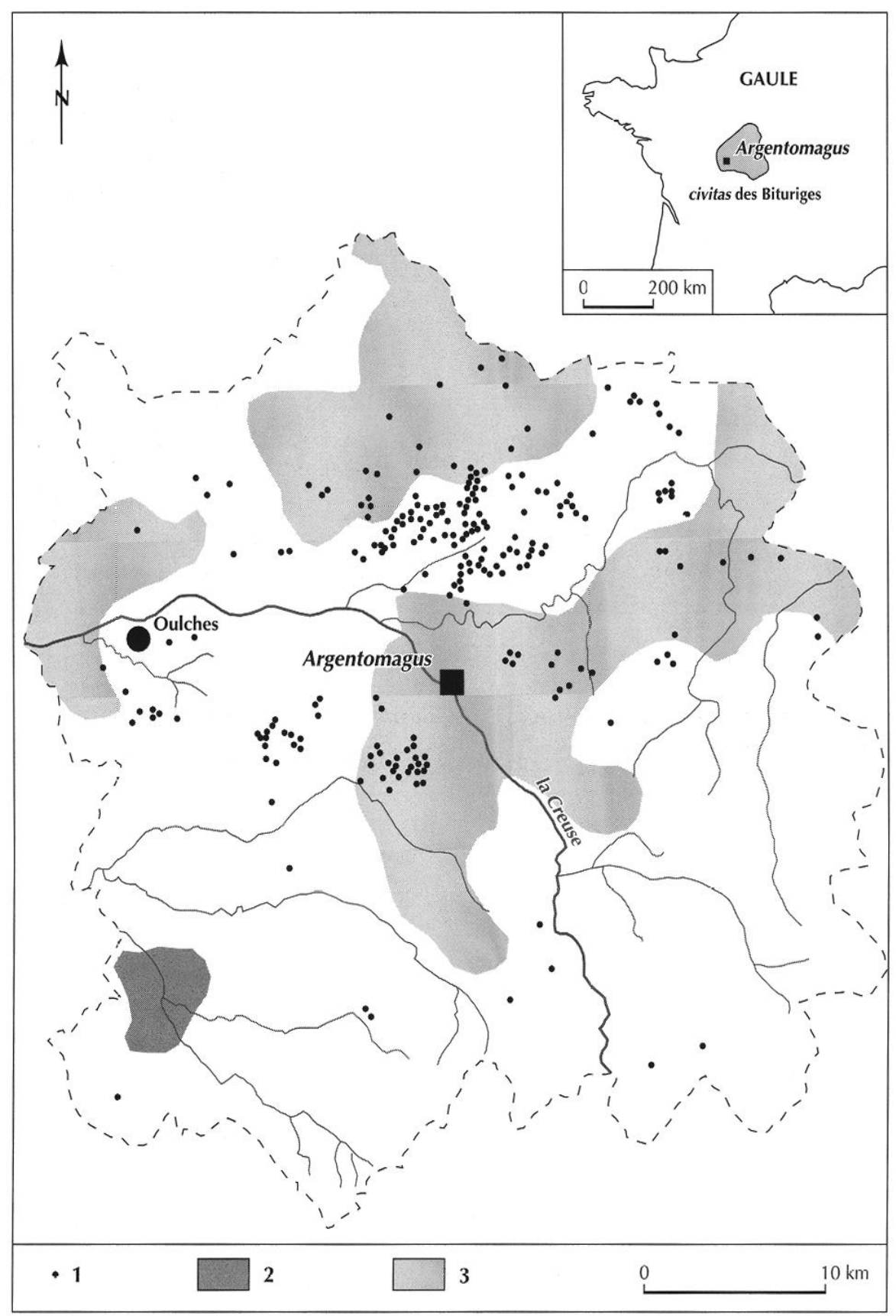

Fig. 25 - Carte de répartition des ferriers et des gisements de minerai de fer autour d'Argentomagus (d'après L. Laüt) : 1, ferrier ; 2 , gisement de minerai de fer de Chaillac; 3, gisement de minerai pisolithique.

L'atelier métallurgique d'Oulches fait partie d'un vaste ensemble de ferriers répartis de manière plutôt irrégulière autour d'Argentomagus, agglomération secondaire de la civitas des Bituriges (fig. 25). Cette civitas, située au coeur de la Gaule, apparaît dans les textes antiques comme une région bien connue pour sa métal- lurgie du fer. Les sources jalonnent la période allant du I ${ }^{\text {er }}$ s. avant J.-C. au Ve s. après J.-C. ${ }^{89}$. Le premier, César

89. F. Dumasy a étudié en détail toutes les sources antiques qui nous sont parvenues; nous empruntons ici l'essentiel de ses conclusions (Dumasy, 1994). 
(La Guerre des Gaules, 7, 22) signale la maîtrise des Bituriges dans le domaine des techniques d'extraction du minerai de fer, lors du siège d'Avaricum, place forte principale des Bituriges Cubi. Puis, au début du $\mathrm{I}^{\mathrm{er}} \mathrm{s}$. de notre ère, Strabon (Géographie, 4, 2, 2) indique qu'il "y a de remarquables ferronneries chez les Pétrocoriens ainsi que chez les Bituriges Cubi». Après un silence de près de quatre siècles, deux autres textes apportent un témoignage de l'importance de l'activité métallurgique au Bas-Empire dans la région. Le premier est un document officiel, la Notitia Dignitatum, qui passe en revue, entre autres, les fabriques d'armes de l'Empire. Pour la Gaule, une fabrique de tous types d'armes (armorum omnium) est mentionnée à Argentomagus (Notitia Dignitatum, Occ., 9, 31). Ce texte, rédigé entre 379 et 437 après J.-C., met sur le même plan des villes importantes comme Autun et Reims et l'agglomération secondaire des Bituriges qu'est Argentomagus, montrant que le choix de cet emplacement relevait sans doute davantage d'une réelle capacité productive de la région que de considérations politiques ou stratégiques. Enfin, la dernière mention antique est le vers d'un poète gallo-romain, Rutilius Namatianus qui raconte les péripéties de son voyage de retour de Rome en 417 (Rutilius Namatianus, De reditu suo, vers 349-354) ; son passage au large de l'île d'Élbe l'incite à évoquer les régions de l'Empire les plus riches en fer, et parmi celles-ci la cité des Bituriges. Il faut noter que ces différents témoignages concernent l'ensemble de la chaîne opératoire, de l'extraction du minerai (César) à la production d'objets finis (Notitia Dignitatum).

La prospection a montré que les ateliers de réduction directe ne sont pas répartis uniformément sur tout le territoire de l'ancien Berry. Deux zones principales ont été mises en évidence : la région d'Allogny, à une quinzaine de kilomètres au nord de Bourges et la région d'Argentomagus, dans l'Indre. Cette dernière a été jusqu'à présent la plus étudiée, puisqu'elle a fait l'objet de prospections répétées pendant plus de cinq ans, certains secteurs ayant même été prospectés systématiquement ${ }^{90}$. Au total, dans un rayon de $20 \mathrm{~km}$ autour de l'agglomération antique, 242 ateliers de réduction ont été repérés au sol, dont 79 seulement ont pu être datés. Ces derniers sont tous gallo-romains, sauf deux probablement en activité à la fin de l'Indépendance gauloise. Cependant, même à l'intérieur de régions de production, la densité

90. Voir note 86 : L. Laüt, op. cit., 1995. des ferriers n'est pas constante puisque sur la rive droite de la Creuse, à $7 \mathrm{~km}$ au nord d'Argentomagus, 108 ferriers ont été recensés sur une surface de $100 \mathrm{~km}^{2}$ seulement (fig. 25).

Le site d'Oulches est situé à $18 \mathrm{~km}$ à l'ouest d'Argentomagus. Il a fait l'objet de prospections aériennes et de prospections au sol. Il se présente sous la forme d'un amas de scories et de terre cendreuse de $6000 \mathrm{~m}^{2}$ de surface et de $1 \mathrm{~m}$ de hauteur maximale, implanté au sommet du coteau qui domine la rive gauche de la Creuse, sur un terrain en légère pente vers le nord. La prospection au sol ayant permis de ramasser du matériel archéologique antique, dont une monnaie du III $^{e} s$. après J.-C., il a été décidé de fouiller cet atelier qui, par ailleurs, semblait assez bien conservé. Comme il ne pouvait être question de décaper mécaniquement la totalité du site, une prospection électromagnétique ${ }^{91}$ a été entreprise en vue de mettre en évidence des zones différenciées au sein de l'atelier et d'orienter le choix de la partie du site à fouiller.

La carte de susceptibilité magnétique montre la présence au nord et au sud du site de deux zones de forte susceptibilité encadrant une zone de faible susceptibilité. La surface fouillée a donc été choisie de manière à recouvrir en partie chacune de ces zones. Elle a été implantée dans la moitié ouest du ferrier car des blocs de grès de grande taille étaient remontés régulièrement à cet endroit par les labours. Le décapage mécanique a révélé une partition du site en trois zones qui se superposent à celles que la prospection électromagnétique avait mises en évidence : au sud, dans la partie haute, on trouve des débris de minerai; au nord, en bas de pente, sont concentrés les scories, les cendres et des débris de charbon de bois. Entre ces deux zones se trouvent les structures de l'atelier: les fours de réduction apparus immédiatement sous la terre arable, mais aussi les foyers de forge et une installation de retraitement d'un certain type de scories.

\section{LE MINERAI DE FER ET SA PRÉPARATION}

La cité des Bituriges possède de nombreux gisements de minerai de fer facilement accessibles. On en distingue

91. Prospection géophysique effectuée par $G$. Ducomet en 1990 (Centre de recherches géophysiques de Garchy, Nièvre). 
habituellement quatre types principaux, d'après la nature des formations géologiques qui les contiennent. Trois types seulement de minerai sont présents dans la région de l'atelier d'Oulches (fig. 25).

- Le minerai des formations sidérolithiques qui appartient au système tertiaire est parfois appelé minerai pisolithique. Son aspect varie suivant les gisements : il se présente souvent sous forme de grains sphériques libres, mais ces grains peuvent parfois être liés par un ciment ferrugineux. Il se trouve dans les argiles qui tapissent la surface des plateaux calcaires du Berry central. Ce minerai a été exploité de manière intensive aux $\mathrm{XVIII}^{\mathrm{e}}$ et $\mathrm{XIX}^{\mathrm{e}}$ s. pour approvisionner les hauts fourneaux de la région.

- Un deuxième gisement, de morphologie très particulière et de surface réduite, est situé dans la commune de Chaillac, à une vingtaine de kilomètres au sud d'Oulches. Son existence est liée à la présence d'une faille à la période hercynienne. Une minéralisation barytique a ensuite réorganisé localement un gisement de minerai de fer déjà présent ${ }^{92}$. On le rencontre sur une surface de quelques kilomètres carrés seulement, à l'extrémité sudouest du département de l'Indre. Exploité pour le fer jusqu'en 1965, ce gisement fournit actuellement une grande partie de la production nationale de barytine. Ce minerai possède quatre faciès principaux, tous très caractéristiques, ce qui permet de distinguer assez facilement par l'aspect le minerai de ce gisement des minerais des autres formations. Les analyses chimiques (DieudonnéGlad, 1994) apportent des informations précieuses pour identifier à coup sûr ce minerai car il se caractérise par une teneur particulièrement faible en cobalt (entre 1 et $30 \mathrm{ppm}$ ) et en nickel (entre 5 et $14 \mathrm{ppm}$ ).

- À ces deux types de minerai de fer bien connus des géologues et figurant sur les cartes, il faut en ajouter un troisième, l'alios. Son étude relève plutôt de la pédologie car il est constitué de croûtes ferrugineuses dues au dépôt des oxydes de fer dissous par les eaux vadoses. Ce dépôt est favorisé par la présence de niveaux imperméables et se produit au-dessus de ceux-ci. À l'échelle géologique, sa formation est extrêmement rapide: quelques centaines à quelques milliers d'années. Il se rencontre sous la semelle de labour au bas des pentes, lorsque le sous-sol est argileux, c'est-à-dire dans une très

92. D. Ninkovic, Étude géologique et métallogénique du bassin de Chaillac, Thèse, Paris, 1961. grande partie du Berry. Il n'existe aucune mention de l'exploitation de cet alios à l'époque moderne.

Ces trois types de minerai ont-ils été utilisés dans l'Antiquité ? À Oulches, sur 12 échantillons de minerai analysés (tabl. I), 7 proviennent avec certitude du gisement de Chaillac (DIE 9306, 9308, 9309, 9310, 9311, 9803, 9804), 2 contiennent un peu trop de nickel (35 et $27 \mathrm{ppm}$ ) pour être attribués à ce gisement (DIE 9206, 9208) et 3 sont probablement du type sidérolithique (DIE 9205, 9207, 9801). L'approvisionnement en minerai de Chaillac est donc bien attesté sur le site, bien que l'atelier soit situé à proximité immédiate de gisements d'alios et de minerai pisolithique (fig. 25). Il faut cependant noter que la consistance friable de ces derniers minerais explique peut-être la difficulté éprouvée pour les identifier lors de la fouille. Par ailleurs, les analyses chimiques effectuées sur les scories d'Oulches montrent que celles-ci se sont formées à partir du mélange du minerai de Chaillac avec d'autres minerais car leur teneur en cobalt surtout (50 à $150 \mathrm{ppm}$ ) et également parfois en nickel (22 à 142 ppm) est beaucoup trop forte, même en tenant compte d'une concentration dans les scories des impuretés contenues dans la gangue du minerai. Cette observation est confirmée par l'analyse de scories provenant de sites métallurgiques situés dans le voisinage immédiat du gisement de Chaillac. Ces scories possèdent, en effet, une teneur en cobalt ( 34 à $52 \mathrm{ppm}$ ) et en nickel ( 5 à $11 \mathrm{ppm}$ ) très faible, compatible avec l'emploi du seul minerai de Chaillac. Il ne semble donc pas que ce soit un obstacle technique à l'utilisation du minerai de Chaillac seul qui ait conduit les métallurgistes d'Oulches à mélanger les minerais dans les fourneaux de réduction. Le mélange de minerais constaté à Oulches témoigne ainsi de la diversité des approvisionnements de l'atelier à courte et à moyenne distance et d'une certaine vitalité des échanges pendant la période de fonctionnement de ce dernier.

La recherche des vestiges des exploitations anciennes de minerai a été également tentée. Même si César signale que les Bituriges " connaissent et emploient tous les genres de galeries souterraines » dans leurs mines de fer (César, La Guerre des Gaules, 7, 22), ces exploitations n'ont pas laissé de traces visibles dans le paysage actuel. Il est possible que celles-ci aient été totalement oblitérées par les travaux d'extraction de l'époque moderne. En effet, des traces de ces travaux récents sont encore visibles un peu partout, essentiellement dans les zones 


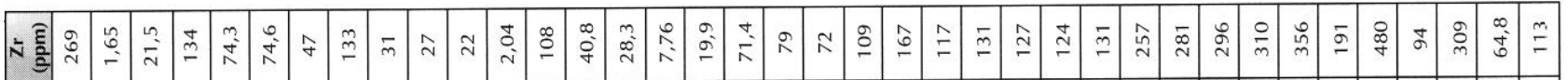

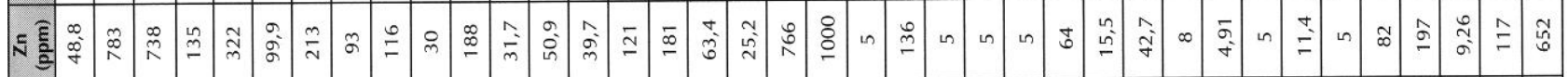

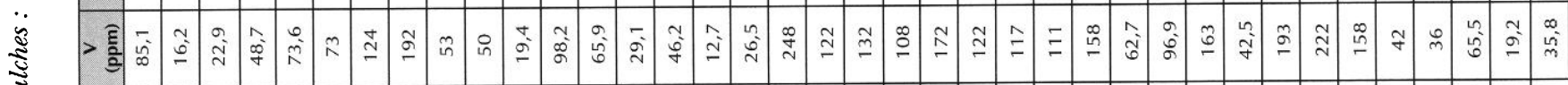

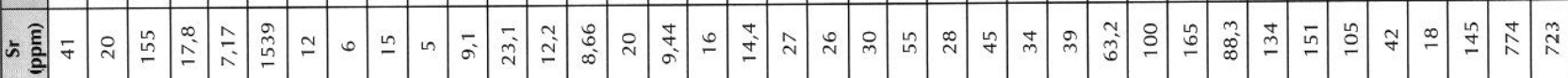

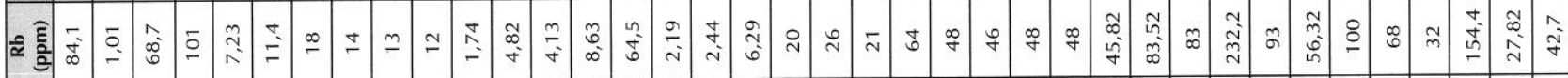

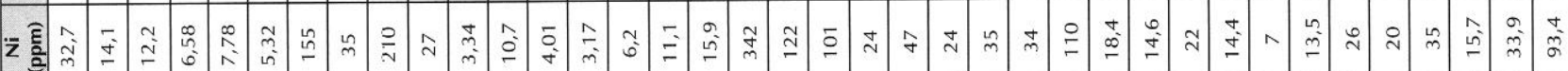
(2)

:

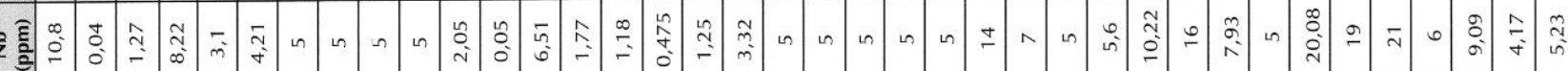

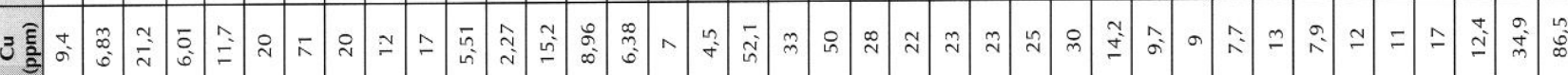
s. $\hat{i} \cdot$ 莡

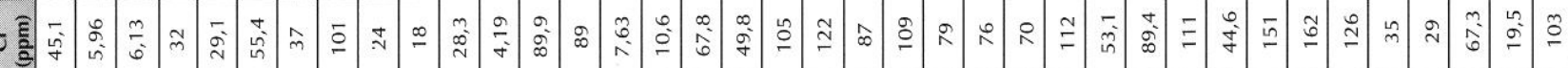

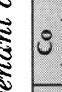

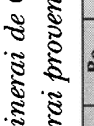

离离 ळ

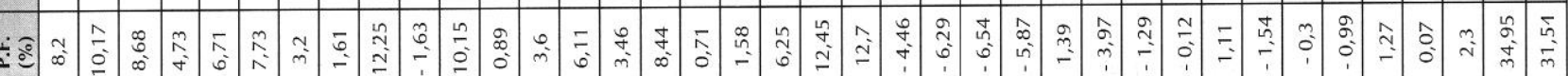

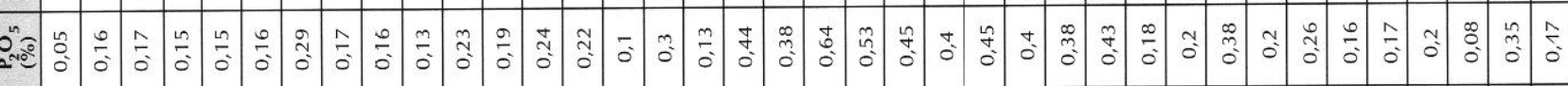

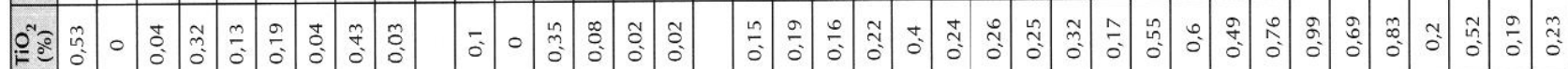
ङ

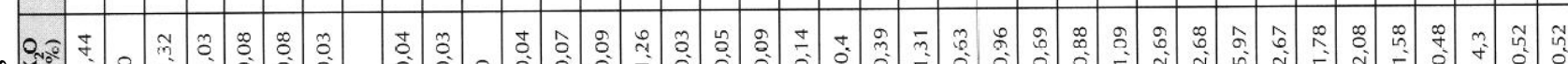

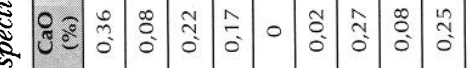

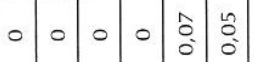

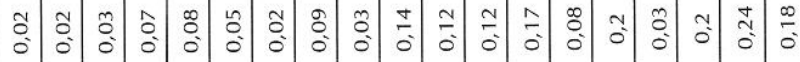


non cultivées. Ce sont des tranchées de quelques dizaines de mètres de longueur et de plusieurs mètres de profondeur ou des entonnoirs de grandes dimensions. Dans les espaces labourés elles ont, en moins d'un siècle, presque complètement disparu et ne restent perceptibles que par la présence de légères dépressions. En ce qui concerne le gisement de Chaillac, nous avons un indice de l'existence d'une exploitation du minerai en mine ou en carrière : un échantillon d'assez grande taille $(20 \mathrm{~cm} \times 10 \mathrm{~cm}$ $\mathrm{x} 10 \mathrm{~cm}$ ), recueilli sur le site d'Oulches, possède une forme anguleuse très marquée, qui ne serait pas explicable si ce morceau avait été prélevé par simple ramassage. Malheureusement les vestiges de ces exploitations anciennes ont sûrement été éliminés par les travaux d'exploitation actuels de la barytine, qui ont lieu en carrière sur une profondeur de plusieurs dizaines de mètres. Il paraît donc vain de rechercher des traces d'exploitations minières antiques dans cette zone.

La présence sur le site métallurgique d'Oulches de couches de couleur rouge, dont les analyses montrent qu'elles sont constituées de fragments de différents minerais réduits en poudre, indique que le minerai subissait un traitement mécanique avant sa réduction. La découverte de plusieurs fragments de meules pourrait laisser à penser que le minerai était broyé avant utilisation. Cependant ces outils n'ont pas été retrouvés en place, mais dans les couches supérieures du site, il est donc délicat d'affirmer que ce sont les instruments utilisés par les métallurgistes.

\section{LA FABRICATION DU FER}

L'atelier s'organise autour de deux fours de réduction du minerai de fer (fig. 26). Le four 1, de forme presque cylindrique, est adossé 'côté ouest à une plate-forme constituée du substrat géologique. Il possède une porte ouverte vers l'est. Son plus grand diamètre intérieur est de 1,70 m. Les parois de ce fourneau sont faites de briques d'argile crue qui se sont rubéfiées lors de l'utilisation du four. Ces briques, grossièrement parallélépipédiques, ont pu être observées dans la couche de démolition. La paroi en place ne montre aucune trace de scorification.

Plusieurs périodes de fonctionnement ont été mises en évidence : après une première série de réductions, le four a subi une réfection générale avec remblaiement du fond du fourneau et reconstruction d'une paroi plaquée contre la paroi existante, côté intérieur du four. Cette opération a emprisonné une couche de charbon de bois entre les deux couches d'argile constituant les états successifs du fond du four. Après une deuxième série de réductions, la structure a, semble-t-il, subi un abandon temporaire, entraînant une désagrégation partielle des parois du four, venant sceller une deuxième couche charbonneuse. Enfin, une troisième phase d'utilisation de ce four de réduction est attestée par la présence d'une épaisse couche cendreuse. L'abandon définitif du four lui succède, suivi par l'écroulement de la paroi dans la cuve.

Accolée au four 1, se trouve une aire de tegulae, posées à l'envers sur un lit de mortier, rebord planté dans le sol. La zone la mieux conservée, au sud, est formée de 10 tuiles. Elle a la forme d'un rectangle de $1,80 \mathrm{~m}$ sur $0,90 \mathrm{~m}$ et possède une bordure d'une vingtaine de centimètres de hauteur formée de petites pierres au sud et à l'ouest, et de blocs de grès plus importants au nord. Des fragments de tuiles sont posés verticalement le long des pierres. Un agencement similaire devait exister immédiatement au nord des gros blocs de grès. Ces aires de tuiles étaient probablement les aires de stockage des matières premières, minerai et charbon de bois, avant leur introduction dans la cheminée du four.

Le four 2 est orienté de la même manière que le four 1 mais possède une structure différente de celui-ci. Pour le construire, le sol géologique a été excavé sur une surface de $5 \mathrm{~m}$ de diamètre et sur $1,30 \mathrm{~m}$ de profondeur. Un massif de blocs de grès liés à l'argile a ensuite été érigé en couronne dans cette excavation sur une largeur de 1,80 m. Le four se trouve au centre de ce massif. La base de la cuve est constituée de l'empilement, sur une soixantaine de centimètres d'épaisseur, de morceaux de parois de four en réemploi. À la sortie du four, un couloir bordé de gros blocs de grès posés verticalement s'étend sur une longueur de 2,50 m. La pierre utilisée est une pierre locale, non taillée. Dans ce four ont été fouillées des couches charbonneuses mêlées de débris de minerai, que la destruction de la partie supérieure du four est venue sceller, comme c'est le cas pour le four 1 . L'accès à la porte du four 2 devait être protégé par un toit soutenu par des poteaux dont les calages ont été retrouvés. Le four débouchait sur une aire de travail dont le sol était également aménagé à l'aide de fragments de paroi de four réemployés. Cette structure semble donc avoir 


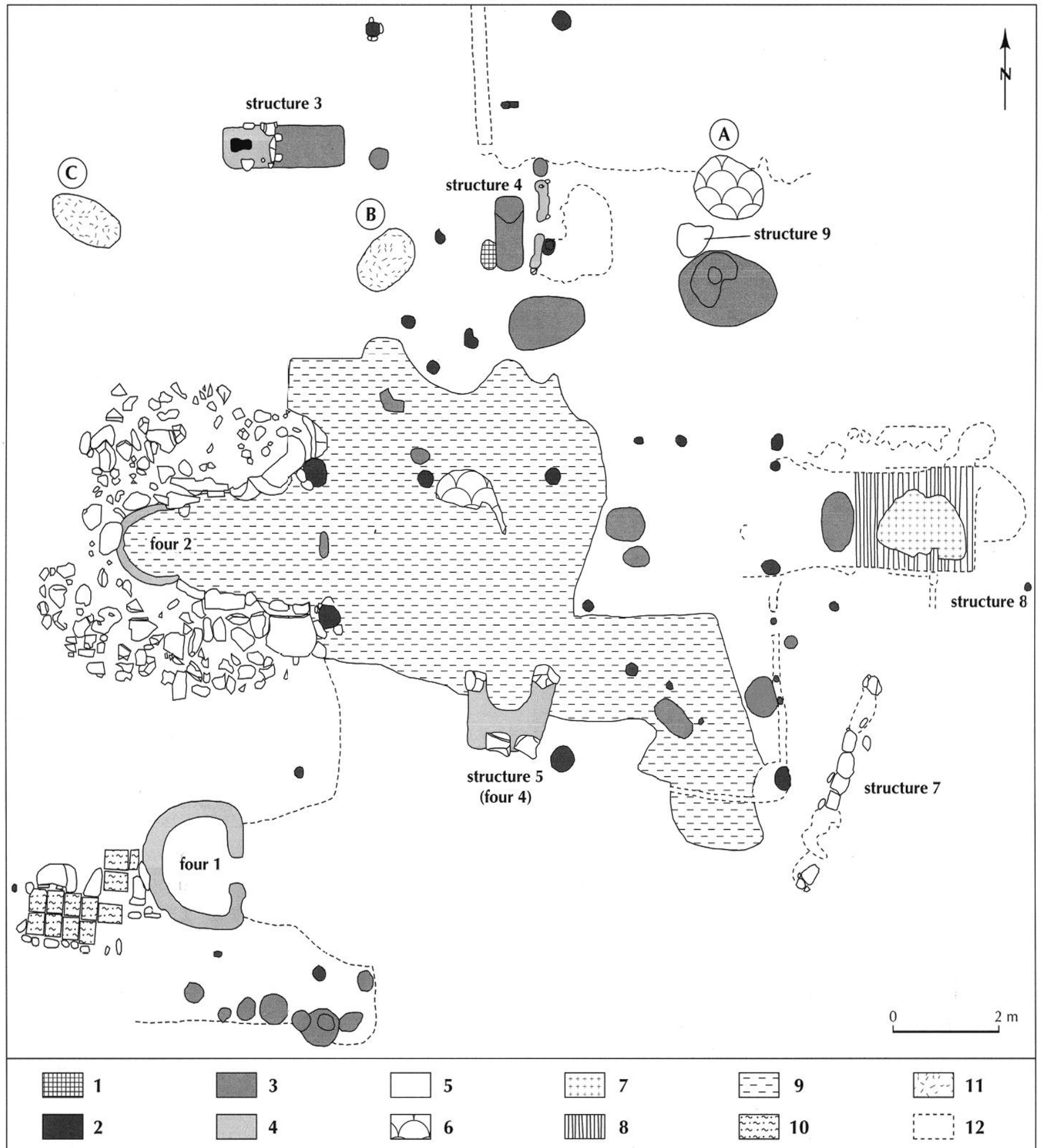

Fig. 26 - Le Latté (Oulches, Indre), plan de l'atelier métallurgique : 1, sol rubéfié ; 2, trous de poteaux; 3, fosses ; 4, structures des fours en argile; 5, pierres ; 6, amas de fragments de métal oxydés; 7, amas de fragments de laitier concrétionné; 8 , empreinte de traverse en bois ; 9, sol fait de fragments de parois de four réemployés ; 10, aire de tuiles ; 11, battitures; 12 , creusement. 


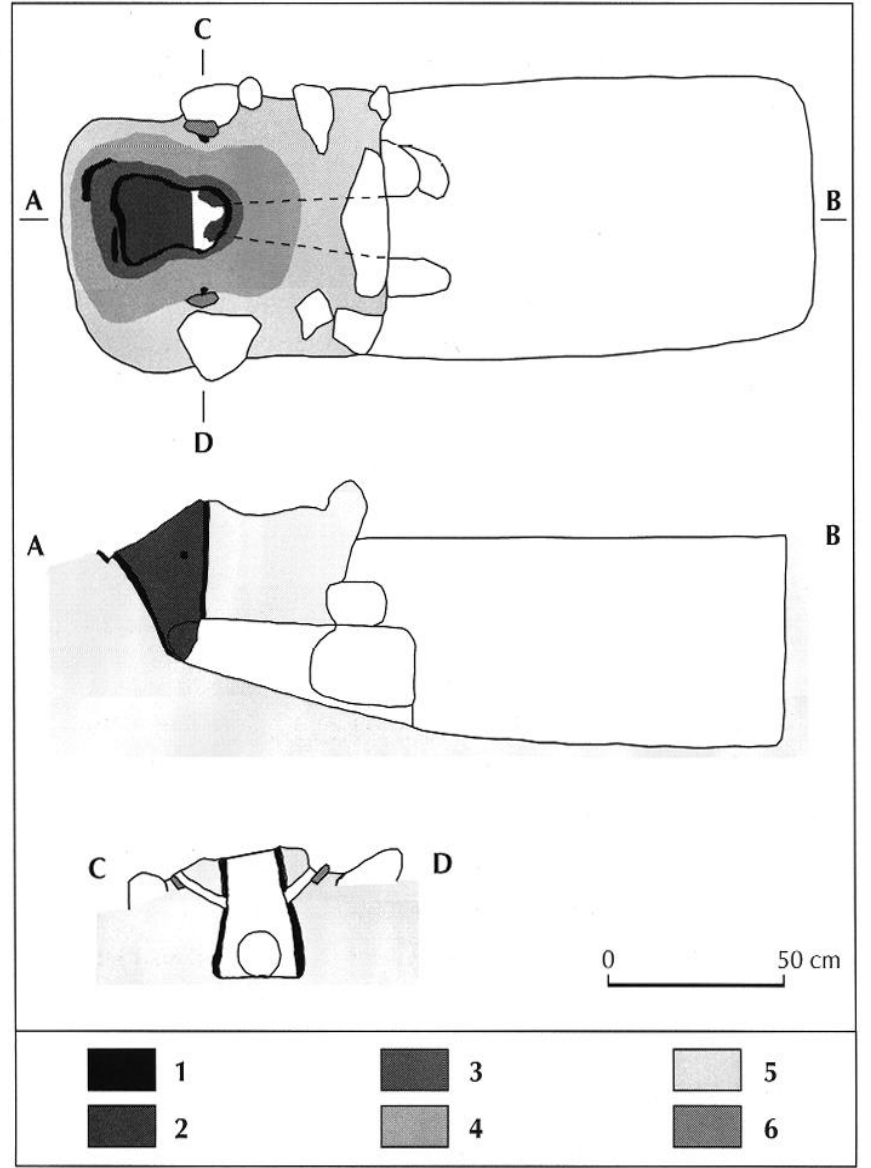

Fig. 27 - Le Latté (Oulches, Indre), plan et coupes du foyer de forge (four 3) : 1 , paroi vitrifiée vue en coupe; 2 , paroi vitrifiée vue de face; 3, paroi de couleur grise (sur la vue en plan); 4, paroi de couleur orange (sur la vue en plan); 5 , paroi de couleur jaune (sur la vue en plan); 6, plaque métallique.

.été construite au cours d'une deuxième phase de fonctionnement de l'atelier, en utilisant des matériaux provenant de la démolition d'autres fourneaux probablement situés hors de l'emprise de la fouille, malgré l'ampleur de la surface traitée $\left(1200 \mathrm{~m}^{2}\right)$.

\section{LES OPÉRATIONS SUCCÉDANT À LA FABRICATION DU MÉTAL : CINGLAGE ET FORGEAGE}

Le lieu où étaient effectuées les étapes succédant à la réduction, cinglage du fer brut et forgeage du métal, se situe au nord des fours de réduction et s'organise autour de deux structures (structures 3 et 4 ). La structure 4 , très abîmée, se compose d'une fosse oblongue de $1,20 \mathrm{~m}$ de longueur et de $0,50 \mathrm{~m}$ de largeur, profonde de $0,30 \mathrm{~m}$. Ses parois ne sont pas vitrifiées, mais seulement rubéfiées par endroits. Le comblement de cette fosse était constitué de sédiment limoneux de couleur brun clair contenant quelques fragments de paroi de four. Cette fosse est bordée à l'est par un muret de terre de $1,50 \mathrm{~m}$ de longueur, de $0,20 \mathrm{~m}$ de largeur et de $0,20 \mathrm{~m}$ de hauteur, interrompu en son milieu sur une largeur de $0,20 \mathrm{~m}$. À l'ouest de la fosse, les sols successifs sont rubéfiés et indurés par la chaleur sur une surface de $0,30 \mathrm{~m}$ par $0,40 \mathrm{~m}$ environ. Ce type de structure est souvent interprété comme foyer de forge comportant un soufflet placé soit sur un petit côté (Domergue dir., 1993, p. 317-321 ; Lebeaupin, 1998, p. 89 et 93), soit au milieu d'un grand côté (Maufras, Fabre, 1998, p. 214). Sur le site de Bram, Aude (Passelac, 1998, p. 131), un culot de forge a même été retrouvé en place au milieu d'un des longs côtés de la fosse, accolé contre la paroi. À Oulches cependant, aucune trace de chauffage n'est visible à l'intérieur de la fosse, seule une partie du sol attenant à celle-ci possède des marques de rubéfaction, comme si des objets portés à haute température y avaient été posés. La structure 4 pourrait alors être le vestige d'un aménagement de foyer de forge construit, comme la structure 3 , qui est beaucoup mieux conservée. Cette dernière se trouve placée directement au nord des deux fourneaux de réduction. Le foyer de forge (four 3) a été construit dans une fosse rectangulaire de $2,10 \mathrm{~m}$ sur $0,80 \mathrm{~m}$ et $0,50 \mathrm{~m}$ de profondeur, creusée dans le substrat géologique, et occupe environ la moitié du volume de celle-ci (fig. 27). La cuve, de très petite taille, mesure $0,40 \mathrm{~m}$ de longueur et $0,30 \mathrm{~m}$ de largeur. L'ensemble des aménagements liés à la ventilation est encore visible. Celle-ci est assurée à deux niveaux : un conduit de $15 \mathrm{~cm}$ de diamètre percé à partir du fond de la fosse débouche à la base du foyer, sur son côté est. Ce conduit ćtait peutêtre pourvu d'une tuyère, bien qu'aucun vestige de ce type d'objet n'ait été retrouvé sur le site. Deux autres conduits, symétriques par rapport à l'axe est-ouest de la structure, de $2 \mathrm{~cm}$ de diamètre, sont percés à travers la paroi à partir du niveau de circulation, selon une pente de $45^{\circ}$ environ par rapport à l'horizontale. Une plaque métallique, sur laquelle venait reposer l'embouchure du soufflet, est visible au débouché extérieur de chaque conduit. Une pierre soutenait le bâti de chacun des soufflets. Ce foyer de forge est construit en argile armée de 
quelques pierres. La paroi présente une couleur différente en fonction de sa distance au centre du foyer: jaune et crue à l'extérieur, elle devient orange, puis grise vers le centre. La partie interne de la paroi, en contact direct avec le feu, est vitrifiée sur une épaisseur de 1 à $2 \mathrm{~cm}$, ce qui indique une température importante, probablement supérieure à $1000{ }^{\circ} \mathrm{C}$, lors du fonctionnement du four. Deux réfections successives ont provoqué une réduction du volume intérieur du foyer. La paroi la plus fortement vitrifiée est située à l'aplomb du canal de ventilation inférieur. La chaleur a fait fondre la paroi qui s'est ensuite solidifiée en deux langues de part et d'autre de l'arrivée du flux d'air. Il est donc probable que la ventilation inférieure ait été une ventilation forcée produite à l'aide d'un soufflet placé au fond de la fosse et actionné avec les pieds ou à l'aide d'un bâton, car l'espace n'est pas assez grand à cet endroit pour qu'une personne puisse prendre place au fond de la fosse à côté du soufflet. Cette technique de ventilation " par en dessous " empêche la formation de scories en forme de calottes au fond du foyer, scories qui sont effectivement totalement absentes du site alors qu'elles sont habituellement reconnues comme un indice majeur pour identifier un atelier de forge.

Un ensemble de couches peut être mis en relation avec ces deux structures. À l'est de la structure 4 (fig. 26), le sédiment est particulièrement charbonneux et contient de petites billes de scories de $0,5 \mathrm{~cm}$ de diamètre environ, plutôt caractéristiques des déchets générés par le martelage de la masse de fer brute. Des fragments de métal informes ont également été ramassés dans cette zone. Ces indices montrent donc la présence, in situ, de l'activité succédant à la réduction du minerai de fer, le cinglage, qui permet d'éliminer par martelage à chaud une grande partie des scories résiduelles piégées dans la masse de fer. Une pierre triangulaire (structure 9, fiy. 26) présentant une légère cupule à sa face supérieure, partiellement enterrée dans le sol et située au milieu de la zone de déchets, pourrait être une enclume.

Entre le four 3 et la structure 4, et à l'ouest du foyer de forge (structure 3, fig. 26), se trouvaient d'autres couches cendreuses contenant une très grande quantité de battitures, plaquettes d'oxydes et de silicates de fer (Dunikowski et al., 1996), détachées de la surface de l'objet lors de son façonnage. Ces éléments de très petite taille tombent sur le sol lors du martelage du fer et se mélangent ensuite au sédiment. Difficilement décelables par un tamisage classique, ils sont, en revanche, sensibles à l'attraction par un aimant. Plusieurs états de cette partie de l'atelier ont été mis en évidence. Les sols successifs, d'épaisseur plus ou moins régulière, sont faits de terre argileuse et sont sujets à de nombreuses recharges, ce qui rend la stratigraphie complexe. Trois états, sans compter les recharges ponctuelles, ont ainsi pu être décelés dans une stratigraphie de $20 \mathrm{~cm}$ d'épaisseur entre les structures 3 et 4 . À chaque réaménagement de l'aire de travail correspond le creusement de trous de poteaux et de fosses. Aucun de ces creusements n'a pu être identifié comme le calage d'un billot d'enclume. Aucun élément stratigraphique ne permet d'associer les couches archéologiques contenant les débris issus du martelage à un des deux foyers de forge en particulier. Seul un raisonnement sur la proximité géographique des différents éléments et sur l'utilisation de l'espace peut permettre de poser quelques hypothèses. La structure 3 , que l'on peut restituer avec un soufflet dans la fosse à l'est et deux soufflets latéraux symétriques au nord et au sud, est plutôt accessible par son côté ouest. C'est donc probablement là que se tenait le forgeron. Les couches de battitures repérées en $\mathrm{C}$ seraient donc à associer au four 3 (fig. 26). Cependant aucun ensemble de trous de poteaux ne permet de délimiter plus exactement l'aire de travail et de déterminer si elle était couverte ou à l'air libre.

L'organisation autour de la structure 4 est plus complexe car le fonctionnement de cet éventuel foyer de forge est plus difficile à restituer. Si l'hypothèse de la présence d'un soufflet à l'est, au milieu du grand côté de la structure, est maintenue et si on prend en compte l'intense rubéfaction du sol au coin sud-ouest de la fosse, l'aire de travail se développerait plutôt à l'ouest de la structure 4 et c'est la zone de battitures B que l'on pourrait associer à ce deuxième foyer de forge (fig. 26). Dans ce cas, la zone A qui regroupe les déchets de cinglage du métal n'est associée à aucun foyer connu. La masse de métal était-elle martelée immédiatement à la sortie du four de réduction? La distance (plus de $15 \mathrm{~m}$ ) entre les fours 1 et 2 et la zone de déchets parait trop importante pour que l'on puisse soutenir cette hypothèse. De même, l'hypothèse d'un réchauffage dans le four 4 (structure 5), dont la fonction est incertaine (voir ci-dessous), ne résiste pas davantage à l'argument de la distance. On peut donc supposer la présence d'un troisième foyer de forge dont les vestiges n'ont pu être décelés à la fouille, à l'est de la zone de déchets A. Peut-être était-il construit 
au-dessus du niveau du sol? Ces trois forges ont-elles fonctionné ensemble ou successivement ? Y avait-il une spécialisation des tâches conduisant à l'installation de plusieurs zones de travail complémentaires? La stratigraphie, comme nous l'avons vu plus haut, ne permet pas d'établir une chronologie relative entre ces différents vestiges. En fait, seules les zones de débris $B$ et $C$ posent un problème puisque les vestiges semblent se rapporter à la même activité, tandis que la zone d'épuration concerne une activité plus en amont dans la chaîne opératoire. Un examen pétrographique comparatif des battitures des zones $\mathrm{B}$ et $\mathrm{C}$ pourrait peut-être apporter des précisions sur l'activité de forge effectuée à chacun de ces endroits.

\section{UNE TECHNIQUE MISE EN ÉVIDENCE POUR LA PREMIÈRE FOIS : \\ LA PRODUCTION ET LE TRAITEMENT DES LAITIERS À GRENAILLE}

Plusieurs autres structures, si elles sont bien identifiables individuellement, ne trouvent pas leur place dans les schémas de fonctionnement des ateliers métallurgiques habituellement étudiés par les archéologues. Elles sont cependant associées de manière indiscutable au travail du fer car elles s'organisent en relation avec les structures précédemment décrites et contiennent, en place, des vestiges liés à l'activité métallurgique. Les structures que nous allons décrire constituent, à ce jour, un unicum parmi les structures sidérurgiques antiques et médiévales de France.

Dans l'axe du four 2 a été fouillé un bassin de $40 \mathrm{~cm}$ de profondeur (structure 8, fig. 26) creusé dans l'argile. Le fond de cclui-ci ćtait garni de traverses en bois non jointives, probablement surmontées d'un plancher qui n'a laissé aucune trace. Les traverses ont, quant à elles, laissé leur empreinte, d'une part dans l'argile du substrat et d'autre part dans une concrétion qui s'est formée au fond du bassin au cours de son utilisation. Cette concrétion cimentée par des oxydes de fer est constituée de fragments de scories de type laitier, c'est-à-dire des scories vitreuses, pauvres en fer, que l'on ne trouve pas, ou très peu, habituellement sur les sites antiques. Or des scories de ce type ont été découvertes de manière systématique dans les remblais au cours de la fouille du site
d'Oulches, en morceaux souvent de la taille du poing. Par contraste, les dimensions des éléments composant la concrétion dépassent rarement $3 \mathrm{~cm}$ dans leur plus grande longueur. De plus, les fragments recueillis dans le bassin présentent tous des cassures vives. Le bassin est alimenté en eau par une canalisation (structure 7, fig. 26) provenant de la zone sud du site, dont un tronçon de $4 \mathrm{~m}$ de longueur est bien conservé. Elle est constituée d'un canal creusé dans l'argile du substrat, protégé par une couverture composée de deux pierres posées verticalement, formant piédroits, sur lesquelles repose une pierre plate horizontale. La couverture de la canalisation a disparu $2 \mathrm{~m}$ avant son arrivée dans le bassin, ce qui a provoqué une érosion presque complète de la structure, si bien que la jonction entre la canalisation et le bassin est difficile à étudier. Il est peu probable que l'eau circulant dans la canalisation provienne d'un ruisseau car le site est à une altitude supérieure à celle des cours d'eau les plus proches. L'eau devait donc être distribuée à partir d'une réserve, réservoir construit, mare ou étang dont l'emplacement n'a pu être déterminé.

La découverte de ce bassin contenant des fragments de laitier conduit à poser le problème de l'utilisation de ce qui n'était jusqu'à présent considéré que comme un déchet (Mahé-Le Carlier et al., 1998). Par référence à des produits des époques moderne et contemporaine, ce matériau peut être qualifié de laitier à grenaille. Il s'agit d'un ensemble biphasé, composé de scorie souvent partiellement vitreuse, très peu riche en fer (laitier) dans laquelle sont incluses de petites billes de métal (grenaille). La situation de ce laitier à grenaille rassemblé en amas au fond du bassin laisse à penser qu'il a fait l'objet d'un traitement volontaire. Dans ce cas, sa production n'est peut-être pas accidentelle, comme nous le supposions jusque-là. Les analyses élémentaires (tabl. I) montrent en effet qu'à Oulches les laitiers ont une teneur en $\mathrm{CaO}(5$ à $20 \%)$ nettement supéricure à celle des scories denses (scories normalement produites dans ce type de fourneaux : 1,64 à 2,65\%). Cette teneur en calcium est impossible à atteindre en utilisant les minerais recueillis sur le site ou en envisageant la contamination de la charge du fourneau par les parois des fours puisque tous ces matériaux sont non calciques $(0,08$ à $0,61 \%$ de $\mathrm{CaO})$. L'hypothèse de l'apport de calcium par le charbon de bois a été également explorée puisque les analyses montrent que les cendres de charbon de bois ont une teneur de 40 à $45 \%$ en $\mathrm{CaO}$ (DIE 9811, 9812). Cependant, le 
taux de cendres du charbon de bois étant de l'ordre de $2 \%$, le calcium représente moins de $1 \%$ de la composition du charbon (98\% de carbone environ). Comme la proportion minerai-charbon de bois constituant la charge du fourneau est habituellement évaluée à $1 \mathrm{~kg}$ de minerai pour $1 \mathrm{~kg}$ de charbon et au maximum à $1 \mathrm{~kg}$ de minerai pour $2 \mathrm{~kg}$ de charbon, l'augmentation de la teneur en calcium dans la scorie du fait du charbon de bois, en admettant même que tout le calcium du charbon passe dans la scorie, ne peut dépasser 1 à $2 \%$. Cette hypothèse ne permet donc pas d'expliquer les teneurs de 5 à $20 \%$ de $\mathrm{CaO}$ des laitiers. Ce calcium ne peut provenir que d'un apport ponctuel et volontaire de calcaire ou de chaux dans la charge du fourncau. Dcs hypothc̀ses peuvent alors être proposées. D'une part, deux types d'opérations pouvaient avoir lieu dans l'atelier, les unes sans ajout de calcaire, produisant des scories denses, les autres avec ajout de calcaire générant exclusivement du laitier. Cette solution est peu vraisemblable compte tenu de la faible proportion de laitier à grenaille produit sur le site par rapport aux scories denses, même si l'on suppose que les petits fragments rejetés hors du bassin après éclatement n'ont pas été tous repérés à la fouille. Une ou deux opérations de réduction avec ajout de calcaire auraient en effet probablement suffi à les produire. D'autre part, le caractère permanent de l'installation de retraitement des laitiers renforce l'autre hypothèse, qui serait l'introduction systématique de calcaire lors d'une phase déterminée de la réduction (peut-être à la fin de l'opération), ce qui conduirait à former lors d'une même réduction une grande quantité de scories denses et une quantité beaucoup plus faible de laitier. L'observation, à l'intérieur d'un bloc de laitier, d'une zone de scorie dense est aussi un argument dans ce sens (Mahé-Le Carlier et al., 1998, photo 4h).

Quelle que soit la manière de le produire, ce laitier qui contient du fer sous forme métallique était ensuite retraité. L'aspect des fragments retrouvés dans le bassin fait penser que celui-ci subissait un éclatement par étonnement dans le bassin d'eau. Cela permettait de libérer les fragments de métal qui étaient ensuite récupérés. Cette hypothèse a été validée en laboratoire grâce à un protocole expérimental ${ }^{93}$. Trois morceaux de laitier

93. J. Charmillon, Étude métallographique des déchets de l'atelier sidérurgique du $I^{p}$ siecle d'Oulches (Indre), Rapport de projet de laboratoire, I.MPM/ENSMA, 1998, inédit. d'une dizaine de centimètres de diamètre provenant du site d'Oulches et d'aspect identique aux fragments découverts dans le bassin (laitier vert) ont été chauffés dans un four de laboratoire. Deux d'entre eux jusqu'à la température de $700{ }^{\circ} \mathrm{C}$, le troisième jusqu'à $1000^{\circ} \mathrm{C}$, températures inférieures à celle qui règne dans un four de réduction (1 200 à $1300{ }^{\circ} \mathrm{C}$ ). Ces trois échantillons ont été trempés à la sortie du four dans un baquet d'eau froide. Le laitier s'est fendu au contact de l'eau sans éclater violemment. Après refroidissement il a été constaté la présence d'une multitude de microfissures dans le matériau rendant l'échantillon friable et effritable à la main, opération qui était complètement impossible auparavant puisque souvent même le marteau est inefficace pour briser les morceaux de laitier à froid. Des éléments métalliques, tantôt informes tantôt sphériques, ont ainsi pu être dégagés facilement de leur enveloppe de laitier. L'étude métallographique de 12 échantillons de ce métal montre qu'il s'agit soit d'un acier fortement carburé (teneur en carbone supérieure à 0,8\%-acier hypereutectoïde) lorsque le fragment est informe, soit même de fonte (à teneur en carbone d'environ 4,3\%) lorsque le fragment est de forme sphérique. Ces observations permettent de proposer d'interpréter les vestiges du bassin comme les témoins d'une production volontaire de métal fortement carburé, récupéré au sein même du laitier après étonnement de celui-ci, soit dès que sa solidification était intervenue à la sortie du four de réduction, soit après réchauffement dans un autre four. Reste la question de la qualité du métal associé aux scories denses. Aucun fragment métallique recueilli sur le site d'Oulches ne peut avec certitude être mis en relation avec la production de scories coulées, mais des expérimentations menées ces dernières années ont abouti à la production à la fois de scories coulées et de fer relativement peu carburé ${ }^{94}$. Généralement, dans ces masses de fer expérimentales se côtoient des zones de fer doux (teneur en carbone inférieure à $0,1 \%$ ) et des zones d'acier hypoeutectoïde (teneur en carbone comprise entre 0,1 et $0,8 \%$ ). Il est donc possible qu'à Oulches ait eu lieu la fabrication volontaire par deux procédés différents, d'une part du fer faiblement carburé, qui représente la majeure partie de la production de l'atelier et,

94. J.-F. Dars, A. Papillault, Les forges de Vulcain, film vidéo, CNRS, 1992. P. Crew, S. Crew, The experimental production of bar iron, in: Mangin éd., 1994, p. 175-176. 
d'autre part, d'une petite quantité d'acier très carburé pouvant servir à des usages très particuliers comme, par exemple, fabriquer des tranchants d'objets. Ce résultat demande évidemment à être complété par d'autres découvertes archéologiques.

\section{LES AUTRES STRUCTURES DU SITE ET LEUR INTERPRÉTATION}

La structure 5 (four 4), dont l'ouverture est au nord, est construite à l'aide de blocs de grès assemblés par de la terre argilo-sableuse. Elle repose sur le sol lié au four 2 ct cst donc postérieure à la construction de ce four. En revanche aucune relation stratigraphique n'a pu être mise en évidence entre ce four et le four 1. L'axe du four 4 est perpendiculaire à l'axe des trois autres fours attestés. Il a donc une position particulière dans l'atelier et son emplacement permet d'émettre l'hypothèse d'un fonctionnement lié à celui du four 2. Deux grands blocs de grès dressés verticalement assurent l'ossature arrière de la structure, tandis que les côtés du foyer sont constitués de blocs de plus petite taille. Sa paroi intérieure en argile a subi trois réfections qui ont entraîné la diminution progressive du volume de la cuve. Aucun orifice de ventilation n'a été repéré. La taille de la cuve, une soixantaine de centimètres de diamètre, est modeste comparée à celle des fours de réduction, mais beaucoup plus importante que celle du foyer de forge. Cette structure, de taille intermédiaire entre les deux types de fours précédemment reconnus, ne semble, en outre, pas avoir été soumise à des températures élevées car ses parois ne sont pas vitrifiées et même quelquefois à peine rubéfiées. Le rôle de ce four dans la chaîne opératoire de l'atelier reste à définir, mais il ne semble s'agir ni d'un four de réduction ni d'un foyer de forge et on peut se demander s'il ne doit pas être associé aux structures de traitement du laitier pour le réchauffage de celui-ci avant éclatement dans le bassin.

De nombreux trous de poteaux et de piquets ont également été découverts. Leur plan ne définit pas très nettement des structures, mais quelques alignements semblent significatifs. À la sortie du four 2, du côté nord, un ensemble de trois trous de poteaux alignés, de diamètre compris entre 30 et $60 \mathrm{~cm}$, définit une limite entre la zone où a lieu la réduction et celle où a lieu le travail de forge. Le trou de poteau le plus grand, situé au pied nord du massif de pierre entourant le four, possède son symétrique côté sud, mais de ce côté aucun alignement de trous de poteaux vers l'est n'a pu être observé. À l'est du four 4 nous avons également rencontré une structure comportant des sablières basses. La sablière basse de direction nord-sud prend appui sur deux poteaux principaux à ses extrémités et est interrompue par deux trous de poteaux de taille inférieure qui peuvent définir un accès. L'autre sablière, de direction générale est-ouest, ne forme pas un angle droit avec la précédente. Elle prend la direction d'un trou de poteau de très grande taille ( $60 \mathrm{~cm}$ de diamètre, $1 \mathrm{~m}$ de profondeur), creusé à l'angle sud-est du four 4. On suit son empreinte dans le sol sur $2 \mathrm{~m}$ environ, puis elle n'est plus perceptible. Un bâtiment était donc probablement accolé au four 4, bâtiment fermé sur deux côtés par un mur en matériaux périssables, ouvert à l'ouest du côté du four 4 et au nord du côté de l'accès au bassin. La fonction de cette structure est difficilement déterminable dans la mesure où le sol était recouvert d'une couche d'occupation très fine ne contenant aucun élément caractéristique, ni d'une activité métallurgique ni d'une activité domestique. Plusieurs fonctions peuvent être envisagées sans qu'elles s'excluent mutuellement : stockage de matériaux (charbon de bois, laitier en attente de traitement, etc.), abris, création d'une zone d'obscurité relative pour mieux estimer la température des matériaux dans le four 4 . Une autre série de trous de poteaux située au nord de cette zone semble prolonger la sablière basse nord-sud. Dans ce cas nous aurions affaire à une construction placée en face du four 2 et commandant l'accès au bassin. De plus de nombreuses fosses parsèment le site, mais ni leur remplissage ni leur position ne nous renseignent sur leur fonction.

\section{DATATION DE L'ATELIER}

Durant la campagne de fouille de 1993 ont été mises au jour 31 monnaies dispersées dans les couches archéologiques, surtout dans la partie sud du site. Toutes ont été émises entre 259 et 294 après J.-C., sauf une monnaie de Constance II datée des années 337-361 ${ }^{95}$. Cette monnaie isolée chronologiquement pouvait apparaître comme

95. Identification J.-L. Roche (Bourges). 
erratique, mais sa position stratigraphique ne justifiait pas un tel traitement. Il semblerait donc que le site d'Oulches ait fonctionné durant le IV ${ }^{\mathrm{c}} \mathrm{s}$. Une telle hypothèse ne contredirait pas, en tout cas, ce que nous savons de la circulation monétaire durant l'Antiquité tardive. L'étude de la céramique confirme cette datation tardive et permet même de proposer un fonctionnement après la seconde moitié du IV ${ }^{e}$ s. ${ }^{96}$. D'autre part, des échantillons de charbon de bois ont été prélevés dans les couches présentes au fond des fours de réduction, afin de dater le fonctionnement de ceux-ci, ainsi que dans une couche charbonneuse voisine du foyer de forge. Ces analyses ${ }^{97}$ ont permis d'obtenir des courbes de probabilités s'étendant du ${ }^{\mathrm{er}} \mathrm{s}$. à la fin du $\mathrm{V}^{\mathrm{e}} \mathrm{s}$. Si l'on part de l'hypothèse d'un fonctionnement simultané des fours de réduction, seule l'intersection des courbes de probabilités est signifiante. Celle-ci recouvre la période 220-380 après J.-C. Cette datation concerne seulement les structures connues. Comme nous l'avons relevé plus haut, le four 2 et le sol attenant utilisent dans leur structure des fragments de paroi de four en réemploi : l'activité métallurgique antérieure à celle que nous essayons de dater n'a pas laissé de vestiges identifiables dans la zone fouillée, qui représente environ $20 \%$ de la surface totale du ferrier.

Une tentative a également été faite pour évaluer la durée de fonctionnement de l'atelier en utilisant la dendrochronologie. En partant de l'hypothèse que le charbon de bois est fabriqué immédiatement après l'abattage des arbres et consommé sans tarder, la datation relative de l'abattage des arbres nous permet de mesurer la durée de l'activité métallurgique sur le site, mais pas de dater cette activité de manière absolue, car les séquences sont trop courtes pour être calées sur les courbes-étalons. Trois séquences d'abattage principales ont été mises en évidence $^{98}$ : une de 26 années consécutives, une autre de 15 années et la troisième de 10 années. Ces séquences ne

96. Étude de la céramique effectuée par Isabelle Bouchain dans le cadre d'une thèse de l'université Paris I sur la céramique gallo-romaine d'Argentomagus et de sa région (soutenance prochaine).

97. Effectuées par Archéolabs sous les références ARC91/R1089C, ARC92/R1154C.

98. En 1991 et 1992 Archéolabs a effectué l'étude dendrochronologique de 236 charbons de bois (ARC91/R1089D et ARC92/R1154D) issus des différentes couches du site. se recouvrent pas, même partiellement, ce qui permet d'émettre deux hypothèses : soit ces séries d'abattages ne sont pas contemporaines, auquel cas le site aurait fonctionné pendant au moins 51 ans avec deux interruptions dont on ne peut évaluer la durée, soit les bois sont contemporains mais issus de secteurs d'écologie différente, ce qui indiquerait un approvisionnement des artisans en différents lieux pour une même campagne de travail. Les éléments manquent pour choisir l'une ou l'autre solution. Quoi qu'il en soit, pour la première fois nous avons accès à des informations sur la durée d'exploitation d'un atelier de réduction du minerai de fer. Nous sommes en effet assurée que le site a été utilisé pendant au moins 26 années consécutives, soit plus d'une génération.

$$
\text { * * }
$$

L'atelier métallurgique d'Oulches est l'un des rares sites en France à avoir livré des structures correspondant à toutes les étapes connues de la métallurgie du fer à l'époque antique : concassage de minerai provenant à la fois de gisements locaux et d'un gisement éloigné d'une vingtaine de kilomètres du site, réduction de ce minerai pour la fabrication de métal, épuration de la loupe de métal par expulsion des dernières scories, forgeage d'objets à partir de ce métal épuré. La découverte d'un bassin contenant des débris de laitier à grenaille a permis, grâce à des analyses et des reconstitutions expérimentales, de mettre en évidence des activités totalement inconnues jusqu'ici : la fabrication volontaire de laitier contenant des fragments métalliques fortement carburés grâce à un ajout de calcaire à la charge du four, et le traitement de ces scories par éclatement à chaud au contact de l'eau pour la récupération des fragments métalliques. Cette pratique est la preuve d'un savoir-faire très élaboré dans la maîtrise de la réduction du minerai, mais aussi dans l'identification des différentes qualités de métal. La durée de fonctionnement de l'atelier en fait un établissement pleinement inséré dans le tissu économique rural de son époque, probablement en relation commerciale avec les exploitations agricoles voisines. Encore en activité à la fin du IVe s. cet atelier métallurgique est peut-être également à mettre en relation avec la fabrique d'armes signalée par la Notitia Dignitatum à Argentomagus, à laquelle il aurait pu fournir une matière première déjà élaborée. 